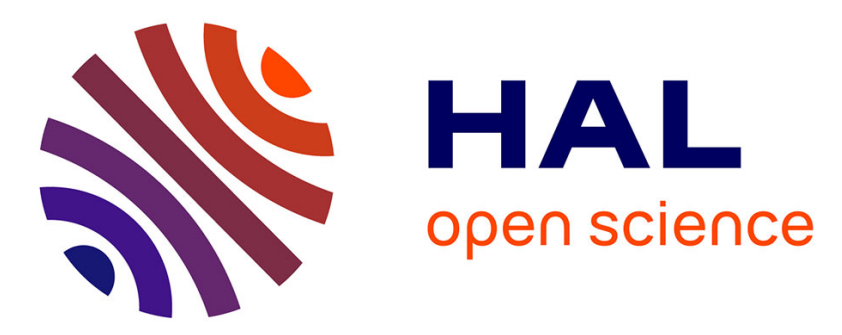

\title{
Global stability for a model of competition in the chemostat with microbial inputs
}

Gonzalo Robledo, Frédéric Grognard, Jean-Luc Gouzé

\section{To cite this version:}

Gonzalo Robledo, Frédéric Grognard, Jean-Luc Gouzé. Global stability for a model of competition in the chemostat with microbial inputs. Nonlinear Analysis: Real World Applications, 2012, 13 (2), pp.582-598. 10.1016/j.nonrwa.2011.07.049 . hal-00848445

\section{HAL Id: hal-00848445 \\ https://hal.inria.fr/hal-00848445}

Submitted on 26 Jul 2013

HAL is a multi-disciplinary open access archive for the deposit and dissemination of scientific research documents, whether they are published or not. The documents may come from teaching and research institutions in France or abroad, or from public or private research centers.
L'archive ouverte pluridisciplinaire HAL, est destinée au dépôt et à la diffusion de documents scientifiques de niveau recherche, publiés ou non, émanant des établissements d'enseignement et de recherche français ou étrangers, des laboratoires publics ou privés. 


\title{
GLOBAL STABILITY FOR A MODEL OF COMPETITION IN THE CHEMOSTAT WITH MICROBIAL INPUTS
}

\author{
GONZALO ROBLEDO, FRÉDÉRIC GROGNARD, AND JEAN-LUC GOUZÉ
}

\begin{abstract}
We propose a model of competition of $n$ species in a chemostat, with constant input of some species. We mainly emphasize the case that can lead to coexistence in the chemostat in a non-trivial way, i.e., where the $n-1$ less competitive species are in the input. We prove that if the inputs satisfy a constraint, the coexistence between the species is obtained in the form of a globally asymptotically stable (GAS) positive equilibrium, while a GAS equilibrium without the dominant species is achieved if the constraint is not satisfied. This work is round up with a thorough study of all the situations that can arise when having an arbitrary number of species into the chemostat inputs: this always results in a GAS equilibrium that either does or does not encompass one of the species that is not present in the input.
\end{abstract}

\section{INTRODUCTION}

The chemostat is a continuous bioreactor with constant volume $V$, which is used to grow microorganisms for experimental and industrial purposes. We will consider the special case where the chemostat contains $n$ species of microorganisms that are in competition for a single limiting substrate. Considering that the substrate is pumped into the chemostat at rate $F>0$ with concentration $s_{i n}>0$ and the mixing of substrate/biomass is pumped out of the chemostat at the same rate, the relationship between the species and the limiting substrate in an homogeneous liquid medium is described by the ODE system [38]:

$$
\left\{\begin{aligned}
\dot{s} & =D\left(s_{i n}-s\right)-\sum_{i=1}^{n} \gamma_{i}^{-1} f_{i}(s) x_{i} \\
\dot{x}_{i} & =x_{i} f_{i}(s)-D x_{i}, \quad i=1, \ldots, n
\end{aligned}\right.
$$

where $s \in \mathbb{R}_{+}$and $x_{i} \in \mathbb{R}_{+}$denote the substrate concentration and the biomass density of the $i$ th species of microorganisms, $D=F / V$ is the dilution rate, the functions $f_{i}(s)$ represent the per capita growth rate of the $i$ th species and $\gamma_{i}>0$ is a yield constant related to the conversion rate of substrate into new biomass. We assume that $f_{i}: \mathbb{R}_{+} \mapsto \mathbb{R}_{+}, D$ and $s_{\text {in }}$ are such that:

(C1) The functions $f_{i}$ are continuously differentiable, increasing and $f_{i}(0)=0$.

(C2) The equation $f_{i}(s)=D$ has one solution $\lambda_{i} \in\left(0, s_{i n}\right)$ for all $i=1, \ldots, n$.

(C3) The species $x_{i}$ are labeled so that:

$$
0<\lambda_{n}<\lambda_{n-1}<\ldots<\lambda_{1}<s_{i n} .
$$

The asymptotic behavior of (1.1) is described by the Competitive exclusion principle [14], [38]: 
Proposition 1 (Competitive exclusion principle, [38]). Assume that (C1)(C3) are satisfied with $x_{n}(0)>0$. Then, the solutions of system (1.1) satisfy:

$$
\lim _{t \rightarrow+\infty}\left(s(t), x_{1}(t), \ldots, x_{n-1}(t), x_{n}(t)\right)=\left(\lambda_{n}, 0, \ldots, 0, \gamma_{n}\left[s_{i n}-\lambda_{n}\right]\right) .
$$

That is, the $n$-th species is the sole successful competitor since it requires the smallest substrate concentration $\lambda_{n}$ to have a growth rate equivalent to the dilution rate $D$, while the other species cannot compete successfully and disappear in the long term. Observe that (C3) implies that, in absence of the originally successful competitor, the $(n-1)$-th species will become the only surviving species.

In this text, we will call $x_{n}$ the dominant or the superior competitor. It is worth noting that this species is not intrinsically dominating the others; this dominant character is related to the choice of $D$ : one species could be successful for one value of $D$ and another one for another value of $D$. In this paper, $D$ and $s_{i n}$ are fixed a priori; there is therefore no problem in terming one species the dominant species. The other species will be termed inferior.

An asymptotic behavior opposite to the extinction is the uniform persistence:

Definition 1. [38] The $i$-th species is uniformly persistent if there exists a constant $\delta_{i}>0$ independent of the initial conditions of (1.1) such that:

$$
x_{i}(0)>0 \Rightarrow \liminf _{t \rightarrow+\infty} x_{i}(t)>\delta_{i} .
$$

In spite of the competitive exclusion having been verified experimentally [8], [13], [36], the uniform persistence is observed in several ecosystems. This duality has stimulated a considerable amount of work in order to be explained [30], [31]. In this context, we can distinguish several approaches:

a) Time variable inputs: models where $s_{i n}, D$ or $\gamma_{i}$ becomes time variable functions. Indeed $\gamma_{i}$ becomes a time variable function in [1], [35]. $D$ is a periodic function in [22], [26], [42] and $s_{i n}$ is a periodic function in [17], [37], [42].

b) Inputs as function of the state variables: models where $D$ becomes a function of the state variables (called a feedback in the framework of control theory) as in $[5],[9],[27]$ (all of them in a two-dimensional framework) and [6], [20] in a threedimensional framework.

c) Heterogeneity of the liquid medium, which was described by using either PDE (see [11], [19], [32], [34]) or gradostat equations (see [15], [33] and references therein).

d) Other approaches: as crowding effects [7], flocculation [12], multi-substrate feeding [11], [23], impulsive input of substrate concentration [28], [29], [44], intraspecific competition [24].

As far as we know, there exists few approaches leading to the coexistence of $n \geq 3$ species (as a stable equilibrium) with constant inputs. In order to tackle that problem, we will propose a modified chemostat model, which receives an input concentration $x_{j}^{0}(j=1, \ldots, n-1)$ for one of each inferior competitor. It will be interesting to point out that, though the introduced species are guaranteed to survive in the chemostat at equilibrium, this strategy does not ensure directly the coexistence of all the species. Indeed, we will prove that there exists a coexistence threshold ensuring the coexistence of all species; above the threshold, the dominant competitor is washed-out of the chemostat. As far as we are aware, this problem has not been previously studied and its proof uses polytopic Lyapunov functions, which have been recently introduced in the literature [10] (for other Lyapunov stability approaches involving piecewise continuous and/or differentiable functions, see [21], 
[41], [43]). Building on the result that we obtain for this chemostat with $n-1$ biomass inputs, we will explore the behaviors that occur when only introducing $n-k$ different biomass densities in the input (for $k>1$ ) and show that only the strongest of the $k$ biomasses that are absent in the input can potentially survive at equilibrium (if there is no superior biomass in the input).

The paper is organized as follows. Section 2 presents our model of competition between $n$ species with $n-1$ inputs, with an equilibrium study and the statement of the two main theorems. Section 3 presents some qualitative properties of the solutions: boundedness, uniform persistence of the inferior competitors, and asymptotic behavior in absence of the superior competitor.

The proof of coexistence of all species (Theorem 1) is given in Section 4: it goes through a proof of persistence of all competitors, a stability analysis on the mass-balance manifold and is concluded by an $\omega$-limit set study for the full system that proves global asymptotic stability of the positive equilibrium. Theorem 2, that describes when extinction takes place in that situation, is proved in Section 5. Finally, these results are used to characterize the general behavior when the number of inputs is different from $n-1$ in Section 6. Finally, some illustrative examples are shown in Section 7 before discussions in Section 8.

\section{Statement of THE MODEL, EQUilibria AND MAIN RESUlts}

The classical competitive exclusion (Proposition 1) implies that the first $n-1$ species cannot survive in the long term. In order to counter that phenomenon, we propose a model where a fixed concentration $x_{i}^{0}>0(i=1, \ldots, n-1)$ of the inferior species is introduced continuously in the chemostat in order to make its uniform persistence possible. The model becomes:

$$
\left\{\begin{aligned}
\dot{s} & =D\left[s^{0}-s\right]-\sum_{i=1}^{n} \gamma_{i}^{-1} f_{i}(s) x_{i}, \\
\dot{x}_{j} & =x_{j} f_{j}(s)+D\left[x_{j}^{0}-x_{j}\right], \quad j=1, \ldots, n-1 \\
\dot{x}_{n} & =x_{n} f_{n}(s)-D x_{n},
\end{aligned}\right.
$$

where $s \in \mathbb{R}_{+}, x \in \mathbb{R}_{+}^{n}$, and $s^{0}>0$ and $x_{j}^{0}>0(j=1, \ldots, n-1)$ are constants. For convenience, we will then define $s_{i n}$ as:

$$
s_{i n}=s^{0}+\sum_{i=1}^{n-1} \gamma_{i}^{-1} x_{i}^{0}
$$

In order to state the main results of this paper, we first need to identify the equilibria that can occur in (2.1). The number of equilibria that can occur as well as their stability depend on a condition that will be central in this paper:

\section{Coexistence condition}

$$
\sum_{i=1}^{n-1} \frac{\gamma_{i}^{-1} D x_{i}^{0}}{D-f_{i}\left(\lambda_{n}\right)}<s_{i n}-\lambda_{n}
$$

This condition can therefore always be satisfied by taking these inputs small enough (under the assumption that $\lambda_{n}<s^{0}$ ). Indeed, this becomes more clear by 
using (2.2) and rewriting (2.3) as follows:

$$
\lambda_{n}+\sum_{i=1}^{n-1} \frac{\gamma_{i}^{-1} D x_{i}^{0} f_{i}\left(\lambda_{n}\right)}{D-f_{i}\left(\lambda_{n}\right)}<s^{0} .
$$

In the sequel, we will use the expression "the coexistence condition is strictly not satisfied" to state that $\sum_{i=1}^{n-1} \frac{\gamma_{i}^{-1} D x_{i}^{0}}{D-f_{i}\left(\lambda_{n}\right)}>s_{i n}-\lambda_{n}$.

Depending on this condition, there can be either one or two equilibria, which are detailed in the following lemma (the - easy- proof is given in the next section):

Lemma 1. Assume that (C1)-(C3) are satisfied, then:

i) System (2.1) has a unique equilibrium in $\partial \mathbb{R}_{+}^{n+1}$ :

$$
\bar{E}=\left(\bar{s}, \bar{x}_{1}, \ldots, \bar{x}_{n-1}, 0\right) \in \partial \mathbb{R}_{+}^{n+1}
$$

with $\bar{x}_{i}$ defined by:

$$
\bar{x}_{i}=\frac{D x_{i}^{0}}{D-f_{i}(\bar{s})} \quad \text { with } \quad i=1, \ldots, n-1,
$$

and $\bar{s} \in\left(0, \lambda_{n-1}\right)$ is the unique fixed point of $\mathcal{G}_{n}:\left[0, \lambda_{n-1}\right) \mapsto \mathbb{R}_{+}$given by:

$$
\mathcal{G}_{n}(s)=s_{i n}-\sum_{j=1}^{n-1} \frac{\gamma_{j}^{-1} D x_{j}^{0}}{D-f_{j}(s)} .
$$

In addition, $\bar{s} \in\left(\lambda_{n}, \lambda_{n-1}\right)$ when (2.3) is satisfied and $\bar{s} \in\left(0, \lambda_{n}\right)$ when it is strictly not satisfied.

ii) System (2.1) has an equilibrium in $\operatorname{Int} \mathbb{R}_{+}^{n+1}$ if and only if (2.3) holds; this equilibrium is unique and is defined as

$$
E^{*}=\left(\lambda_{n}, x_{1}^{*}, \ldots, x_{n}^{*}\right) \in \operatorname{Int} \mathbb{R}_{+}^{n+1}
$$

with $x_{i}^{*}$ defined by:

$$
\begin{aligned}
& x_{i}^{*}=\frac{D x_{i}^{0}}{D-f_{i}\left(\lambda_{n}\right)} \quad \text { for } \quad i=1, \ldots, n-1, \\
& x_{n}^{*}=\gamma_{n}\left\{s_{i n}-\lambda_{n}-\left(\gamma_{1}^{-1} x_{1}^{*}+\ldots+\gamma_{n-1}^{-1} x_{n-1}^{*}\right)\right\},
\end{aligned}
$$

iii) If the equality:

$$
\sum_{i=1}^{n-1} \frac{\gamma_{i}^{-1} D x_{i}^{0}}{D-f_{i}\left(\lambda_{n}\right)}=s_{i n}-\lambda_{n}
$$

is satisfied, then $\bar{E}=E^{*}=\left(\lambda_{n}, x_{1}^{*}, \ldots, x_{n-1}^{*}, 0\right)$.

Two facts can be drawn from this proof. The first one is that Condition (2.3) simply is a condition of existence of a positive equilibrium based on the positiveness of $x_{n}^{*}$. The second one is that, based on the expression (2.6) of the $x_{i}^{*}$, Condition (2.3) can be rewritten as

$$
\sum_{i=1}^{n-1} \gamma_{i}^{-1} x_{i}^{*}<s_{i n}-\lambda_{n} .
$$

The asymptotic behavior of (2.1) will be summarized in the following results: 
Theorem 1 (Coexistence of $n$ species). Assume that (C1)-(C3) are satisfied. If the Coexistence Condition (2.3) is satisfied then $\bar{E}$ and $E^{*}$ defined in Lemma 1 are the two equilibria of (2.1) in $\mathbb{R}_{+}^{n+1}$, and all solutions of (2.1) with initial condition in $\mathbb{R}_{+}^{n+1}$ satisfying $x_{n}(0)>0$ converge to the stable equilibrium $E^{*}$ while convergence takes place to $\bar{E}$ if $x_{n}(0)=0$.

Theorem 2 (Extinction of superior competitor). Assume that (C1)-(C3) are satisfied. If the Coexistence Condition (2.3) is strictly not satisfied then $\bar{E}$ defined in Lemma 1 is a globally asymptotically stable equilibrium of (2.1) in $\mathbb{R}_{+}^{n+1}$.

These results ensure that convergence to an equilibrium always takes place, and give a necessary and sufficient condition for coexistence of all species at the equilibrium. Condition (2.3) ensures that, as long as the dominant species is present at the onset, coexistence of all species is achieved through a globally asymptotically stable positive equilibrium. Whenever the positive equilibrium exists it is stable; as soon as it disappears, stability is transferred to the lone equilibrium on the boundary. Theorem 2 indicates that it is not enough to introduce the inferior species in the medium to ensure coexistence; one must make sure that their density is not too high which could wash-out the dominant species.

\section{Fundamental properties of (2.1)}

In this section, we will give, after the proof concerning the equilibria, two results that are instrumental in the proofs of Theorems 1 and 2 but are not specific to one or the other situation: the boundedness of the solutions and the persistence of the inferior species. Also, we will detail what occurs on the lone invariant face of $\mathbb{R}_{+}^{n+1}$, the face where $x_{n}=0$ (and $s, x_{i} \geq 0$ for $i \in\{1, \cdots, n-1\}$ ); the faces where $x_{n}>0$ and some other $x_{i}=0$ are not invariant and the aforementioned persistence result ensures that they are not critical in the study of the model.

Proof of Lemma 1. Assumptions (C2)-(C3) imply that $\dot{x}_{n}=0$ if $s=\lambda_{n}<s_{i n}$ and/or $x_{n}=0$. The two equilibria will be generated by assuming one or the other.

Proof of statement i): We assume that $x_{n}=0$. Notice that $\dot{x}_{i}=0$ (for $i=$ $1, \ldots, n-1)$ and $\dot{s}+\sum_{i=1}^{n-1} \gamma_{i}^{-1} \dot{x}_{i}=0$ if and only if $s$ and $x_{i}$ satisfy the equations

$$
\begin{gathered}
x_{i}=\frac{D x_{i}^{0}}{D-f_{i}(s)} \text { for } i=1, \ldots, n-1, \quad \text { and } \\
\sum_{i=1}^{n-1} \gamma_{i}^{-1} D\left(x_{i}^{0}-x_{i}\right)+D\left(s^{0}-s\right)-\gamma_{n}^{-1} f_{n}(s) x_{n}=0 .
\end{gathered}
$$

By noting that $x_{n}=0$, coupling these equations and using (2.2), it follows that $s$ must be a fixed point of $\mathcal{G}_{n}(\cdot)$ defined in (2.5). Analyzing (2.5), we notice that (C1) implies that $\mathcal{G}_{n}(\cdot)$ is continuous and strictly decreasing in $\left[0, \lambda_{n-1}\right)$. Consequently, the existence of the unique fixed point $\bar{s} \in\left(0, \lambda_{n-1}\right)$ follows from the inequalities:

$$
\mathcal{G}_{n}(0)=s_{0}>0 \text { and } \lim _{s \rightarrow \lambda_{n-1}^{-}} \mathcal{G}_{n}(s)=-\infty .
$$

Evaluating $\mathcal{G}_{n}\left(\lambda_{n}\right)$ then yields

$$
\mathcal{G}_{n}\left(\lambda_{n}\right)=s_{i n}-\sum_{j=1}^{n-1} \frac{\gamma_{j}^{-1} D x_{j}^{0}}{D-f_{j}\left(\lambda_{n}\right)} .
$$


and we can conclude that if $(2.3)$ is satisfied then $\mathcal{G}_{n}\left(\lambda_{n}\right)>\lambda_{n}$. This fact, combined with (3.1) implies that $\bar{s} \in\left(\lambda_{n}, \lambda_{n-1}\right)$. Similarly, if (2.3) strictly does not hold it follows that $\mathcal{G}_{n}\left(\lambda_{n}\right)<\lambda_{n}$ and it can be deduced that $\bar{s} \in\left(0, \lambda_{n}\right)$.

Proof of statement ii): We assume that $s=\lambda_{n}$. Hence, for all $i=1, \ldots, n-1$, $\dot{x}_{i}=0$ if and only if $x_{i}=x_{i}^{*}$. Observe that (C2)-(C3) imply that $x_{i}^{*}>0$ $(i=1, \ldots, n-1)$.

Furthermore, it follows from $\dot{s}+\sum_{i=1}^{n} \gamma_{i}^{-1} \dot{x}_{i} / D=0$ at equilibrium that:

$$
\left[s^{0}-s^{*}\right]+\sum_{i=1}^{n-1} \gamma_{i}^{-1}\left[x_{i}^{0}-x_{i}^{*}\right]-\gamma_{n}^{-1} x_{n}^{*}=0 .
$$

By using $s^{*}=\lambda_{n}$ combined with (2.2) and isolating $x_{n}^{*},(2.7)$ is obtained. Its positiveness is verified by substituting $x_{i}^{*}$ from (2.6) into (2.7) and using (2.3).

Proof of statement iii): The proof is straightforward and is left for the reader.

3.1. Boundedness of solutions. We first recall a classical mass-balance result for chemostats with identical removal rate for all species and the substrate:

Lemma 2. The solutions of (2.1) converge (when $t \rightarrow+\infty)$ to the set:

$$
\Upsilon=\left\{\left(s, x_{1}, \ldots, x_{n}\right) \in \mathbb{R}_{+}^{n+1}: s+\sum_{i=1}^{n} \gamma_{i}^{-1} x_{i}=s_{i n}\right\} .
$$

Proof. By using the change of variables

$$
v=s+\sum_{i=1}^{n} \gamma_{i}^{-1} x_{i}
$$

combined with (2.1)-(2.2), it follows that $v$ satisfies:

$$
\dot{v}=-D v+D s_{i n}
$$

and the Lemma follows.

This lemma allows us to prove stability through the analysis of dynamics on the asymptotic manifold where $v=s_{i n}$, followed by some $\omega$-limit set argument that leads to a stability result for the system in the whole state-space. Also, it shows boundedness of the solutions:

Remark 1. A consequence of Lemma 2 is the existence of a positively invariant compact set $\Omega \subset \mathbb{R}_{+}^{n+1}$ such that any solution of (2.1) enters $\Omega$ in finite time and stays inside $\Omega$ for all subsequent times.

3.2. Uniform persistence of the inferior species. Since they are constantly fed into the chemostat and since the only processes they are involved into are growth and removal at the same rate they are fed, the persistence of the inferior species is to be expected. In the following proof, we show that their density eventually becomes asymptotically larger than $x_{i}^{0}$.

Lemma 3. Species $i$ ( for $i=1, \ldots, n-1)$ is uniformly persistent.

Proof. It is straightforward from model (2.1) that $\dot{x}_{i} \geq D\left(x_{i}^{0}-x_{i}\right)$ with $x_{i}(0)>0$. By comparison results, it follows that $\liminf _{t \rightarrow+\infty} x_{i}(t) \geq x_{i}^{0}$ and the Lemma follows. 
3.3. Asymptotic behavior in absence of dominant competitor. We have just shown that the inferior competitors would be persistent and the main question that Theorems 1 and 2 answer concerns the evolution of $x_{n}$. As a preamble to the proofs of these theorems, it is useful to study what is happening in the absence of $x_{n}$ whose density, since $x_{n}$ is not fed into the chemostat, stays equal to 0 if $x_{n}(0)=0$. Observe that the restriction of $(2.1)$ to the set of initial conditions

$$
\Gamma_{n}=\left\{\left(s, x_{1}, \ldots, x_{n}\right) \in \mathbb{R}_{+}^{n+1}: s \geq 0, x_{i} \geq 0 \quad(i \leq n-1) \quad \text { and } \quad x_{n}=0\right\}
$$

leads to the subsystem:

$$
\left\{\begin{array}{l}
\dot{s}=D\left[s^{0}-s\right]-\sum_{i=1}^{n-1} \gamma_{i}^{-1} f_{i}(s) x_{i}, \\
\dot{x}_{j}=x_{j} f_{j}(s)+D\left[x_{j}^{0}-x_{j}\right], \quad j=1, \ldots, n-1,
\end{array}\right.
$$

with, $s(0), x_{j}(0) \geq 0$.

In addition, by using (3.2), system (3.5) becomes:

$$
\left\{\begin{array}{l}
\dot{v}=D s_{i n}-D v \\
\dot{x}_{j}=x_{j} f_{j}\left(v-\sum_{i=1}^{n-1} \gamma_{i}^{-1} x_{i}\right)+D\left[x_{j}^{0}-x_{j}\right], \quad j=1, \ldots, n-1
\end{array}\right.
$$

with $x_{j}(0) \geq 0$ and $v(0)-\sum_{i=1}^{n-1} \gamma_{i}^{-1} x_{i}(0)=s(0) \geq 0$.

Hence, by Lemma 2, the asymptotic behavior of (3.6) can be described by:

$$
\dot{x}_{j}=x_{j} f_{j}\left(s_{i n}-\sum_{i=1}^{n-1} \gamma_{i}^{-1} x_{i}\right)+D\left[x_{j}^{0}-x_{j}\right], \quad j=1, \ldots, n-1
$$

with initial conditions in the compact set

$$
\mathcal{D}_{n-1}=\left\{\left(x_{1}, \ldots, x_{n-1}\right) \in \mathbb{R}_{+}^{n-1}: \sum_{i=1}^{n-1} \gamma_{i}^{-1} x_{i} \leq s_{i n}\right\} .
$$

Lemma 4. The point $\bar{x}=\left(\bar{x}_{1}, \ldots, \bar{x}_{n-1}\right)$, with $\bar{x}_{i}$ defined by (2.4), is a GAS stable equilibrium of (3.7) with respect to all initial conditions in $\mathcal{D}_{n-1}$.

Proof. Let $x=\left(x_{1}, \ldots, x_{n-1}\right)$ be a solution of $(3.7)$ and notice that $\mathcal{D}_{n-1}$ is positively invariant under the semiflow defined by system (3.7).

Let us define the function $S: \mathcal{D}_{n-1} \mapsto \mathbb{R}$ as follows:

$$
S(x)=\sum_{i=1}^{n-1} \gamma_{i}^{-1}\left(x_{i}-\bar{x}_{i}\right)
$$

Furthermore, let us define the functions (for $i=1, \ldots, n-1$ ):

$$
S_{i}^{+}\left(x_{i}\right)=\max \left\{\gamma_{i}^{-1}\left(x_{i}-\bar{x}_{i}\right), 0\right\}, \quad \text { and } \quad S_{i}^{-}\left(x_{i}\right)=\max \left\{\gamma_{i}^{-1}\left(\bar{x}_{i}-x_{i}\right), 0\right\},
$$

which allow for the definitions of:

$$
S^{+}(x)=\sum_{i=1}^{n-1} S_{i}^{+}\left(x_{i}\right) \geq 0 \quad \text { and } \quad S^{-}(x)=\sum_{i=1}^{n-1} S_{i}^{-}\left(x_{i}\right) \geq 0 .
$$


It is straightforward to verify that $S(x)=S^{+}(x)-S^{-}(x)$. In addition, by using the definition of $S(x)$ we have that

$$
s_{i n}-\sum_{i=1}^{n-1} \gamma_{i}^{-1} x_{i}=s_{i n}-\sum_{i=1}^{n-1} \gamma_{i}^{-1} \bar{x}_{i}-S(x)=\bar{s}-S(x) .
$$

the last equality coming from $\bar{v}=s_{i n}$ when studying (3.7).

Let us define the function $V: \mathcal{D}_{n-1} \mapsto \mathbb{R}_{+}$as follows:

$$
V(x)=\max \left\{S^{+}(x), S^{-}(x)\right\} .
$$

Notice that $V$ is continuous, positive for all $x \in \mathcal{D}_{n} \backslash \bar{x}$ and $V(\bar{x})=0$ as shown in [10]. Note that though the definition of the Lyapunov function looks intricate, it yields a function whose level sets are simple polytopes (See Figure 1).

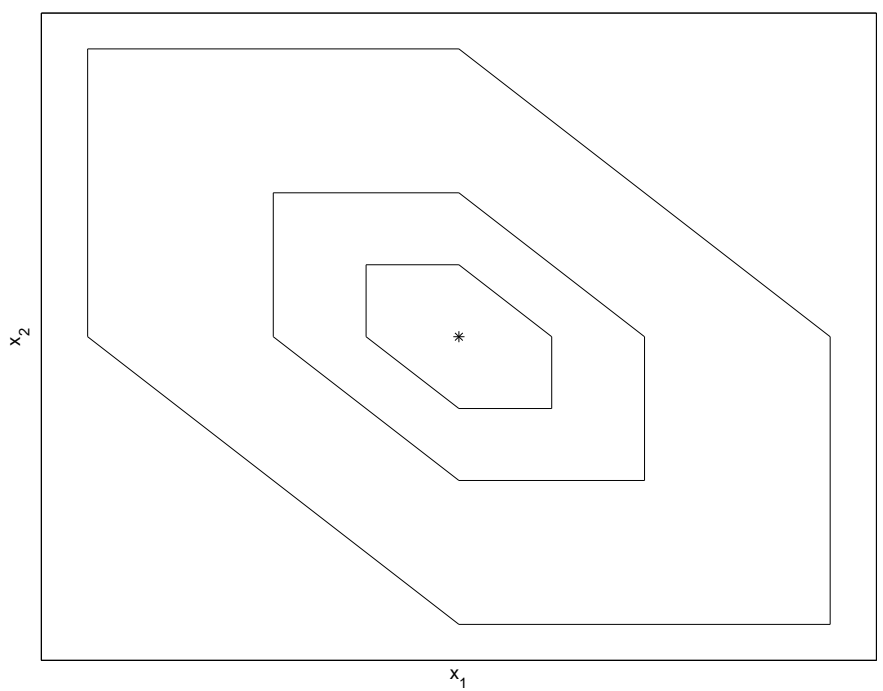

Figure 1. Illustration of level sets of the polytopic Lyapunov function $V(x)$ for $n=2$. This function is continuous and nondifferentiable at the angles and has a minimum at the equilibrium.

Because of the composite structure of $V$, the stability analysis will be separated in several cases:

Case a) $S^{+}(x) \geq S^{-}(x)$ : In this region, we have that $S(x) \geq 0$ and $V(x)=S^{+}(x)$. Equality (3.8) then implies:

$$
\dot{x}_{i}=x_{i} f_{i}(\bar{s}-S(x))+D x_{i}^{0}-D x_{i} \quad \text { for any } \quad i=1, \ldots, n-1 .
$$

Notice that for any $i$ such that

$$
x_{i}(t)>\bar{x}_{i}=\frac{D x_{i}^{0}}{D-f_{i}(\bar{s})},
$$

we have that $D x_{i}^{0}-D x_{i}(t)<-x_{i}(t) f_{i}(\bar{s})$ so that $\dot{x}_{i}$ becomes

$$
\dot{x}_{i}(t)<x_{i}(t)\left\{f_{i}(\bar{s}-S(x(t)))-f_{i}(\bar{s})\right\} \leq 0
$$


where the last equality comes from $S(x(t)) \geq 0$ combined with (C1) and we can conclude that the map $t \mapsto S_{i}^{+}\left(x_{i}(t)\right)=\gamma_{i}^{-1}\left(x_{i}-\bar{x}_{i}\right)$ is decreasing.

Hence, we have proved that if $x_{i}(t)>\bar{x}_{i}$, then $x_{i}$ contributes a decreasing term to $V(x(t))$. On the other hand, if $x_{i}(t)<\bar{x}_{i}$, then $x_{i}$ does not contribute to $V(x(t))$ since $S_{i}^{-}\left(x_{i}(t)\right)=0$. If $x_{i}(t)=\bar{x}_{i}$, a reasoning similar to the one we just held shows that $\dot{x}_{i} \leq 0$, so that $S_{i}^{+}\left(x_{i}(t)\right)$ stays constant at 0 also.

Observe that there exists at least one index $i$ satisfying $x_{i}(t)>\bar{x}_{i}$. Indeed, otherwise we would have $S^{+}(x(t))=0$ and, since $S(x(t))=S^{+}(x(t))-S^{-}(x(t)) \geq$ 0 , it follows that $S^{-}(x(t))=0$, which implies that $x(t)=\bar{x}$ because $S^{+}(x)=$ $S^{-}(x)=0$ imposes that there is no term larger/shorter than $\bar{x}_{i}$. In consequence, it follows that if $V(x)=S^{+}(x(t))$ and $x(t) \neq \bar{x}$, the map

$$
t \mapsto S^{+}(x(t))=\sum_{i=1}^{n-1} S_{i}^{+}\left(x_{i}(t)\right)
$$

is decreasing because there is always some $i$ such that $x_{i}>\bar{x}_{i}$.

Case b) $S^{+}(x) \leq S^{-}(x)$ : By following the lines of the previous case, it can be proved that in this region, the composite map

$$
t \mapsto S^{-}(x(t))=\sum_{i=1}^{n-1} S_{i}^{-}\left(x_{i}(t)\right)
$$

is decreasing as long as $x(t) \neq \bar{x}$.

Summing up these two cases, the map

$$
t \mapsto V(x(t))=\max \left\{S^{+}(x(t)), S^{-}(x(t))\right\}
$$

is always decreasing when $x(t) \neq \bar{x}$ and the Lemma follows by using Lyapunov's Theorem.

Remark 2. This function has been also employed (see [10]) to prove the global asymptotical stability of a positive equilibrium in a model of intra-specific competition in a chemostat.

Remark 3. We have just shown asymptotic stability of $s_{\text {in }}$ for the $\dot{v}$ dynamics (3.3) in Lemma 2 and of $\left(\bar{x}_{1}, \ldots, \bar{x}_{n-1}\right)$ for the $\dot{x}$ dynamics (3.7) in Lemma 4 . Hence, by Theorem 3.2 from [40] it follows that $\left(s_{i n}, \bar{x}_{1}, \ldots, \bar{x}_{n-1}\right)$ is a locally asymptotically stable (LAS) equilibrium of (3.6).

Proposition 2 (Asymptotic behavior in absence of superior competitor). The point $\bar{E}=\left(\bar{s}, \bar{x}_{1}, \ldots, \bar{x}_{n-1}, 0\right)$ is a globally asymptotically stable equilibrium of system (2.1) with respect to all initial conditions in the set $\Gamma_{n}$ defined in (3.4).

Proof. We will prove an equivalent statement: the global stability of the equilibrium $\bar{E}_{v}^{n}=\left(s_{i n}, \bar{x}_{1}, \ldots, \bar{x}_{n-1}\right)$ of $(3.6)$ : let $\left(v_{0}, x_{0}\right) \in \mathbb{R}_{+} \times \mathbb{R}_{+}^{n-1}$ an initial condition of (3.6) satisfying $v_{0}-\sum_{i=1}^{n-1} \gamma_{i}^{-1} x_{0 i} \geq 0$. Denote by $\phi_{t}\left(v_{0}, x_{0}\right)$ the corresponding semiflow (3.5). The $\omega$-limit set of $\left(v_{0}, x_{0}\right)$ is defined by:

$$
\omega\left(v_{0}, x_{0}\right)=\left\{(\tilde{v}, \tilde{x}) \in \mathbb{R}_{+} \times \mathbb{R}_{+}^{n-1}: \exists t_{n} \rightarrow+\infty \lim _{n \rightarrow+\infty} \phi_{t_{n}}\left(v_{0}, x_{0}\right)=(\tilde{v}, \tilde{x})\right\} .
$$


This $\omega$-limit set is non empty because the trajectories are bounded. Given any $(\tilde{v}, \tilde{x}) \in \omega\left(v_{0}, x_{0}\right)$, Lemma 2 implies that $\tilde{v}=s_{\text {in }}$ and by using invariance of $\omega\left(v_{0}, x_{0}\right)$ it follows that $\phi_{t}\left(s_{i n}, \tilde{x}\right) \in \omega\left(v_{0}, x_{0}\right)$ for any $t \geq 0$.

In addition, we have that the $x$ component of $\phi_{t}\left(s_{i n}, \tilde{x}\right) \in \omega\left(v_{0}, x_{0}\right)$ is a solution of (3.7) with initial condition $x(0)=\tilde{x}$. By letting $t \rightarrow+\infty$, it follows by Lemma 4 that $\bar{E}_{v}^{n} \in \omega\left(v_{0}, x_{0}\right)$ and in consequence, there exists a divergent sequence $\left\{t_{k}\right\}_{k}$ such that $\phi_{t_{k}}\left(v_{0}, x_{0}\right)$ converges toward $\bar{E}_{v}^{n}$.

Finally, by Remark 3 we know that $\bar{E}_{v}^{n}$ is also LAS stable, which implies that after some finite time $t_{k}$, the orbit $\phi_{t_{k}}$ enters the basin of attraction of $\bar{E}_{v}^{n}$ and the Proposition follows.

Through this Proposition, we have shown what occurs when $x_{n}(0)=0$ both when the Coexistence Condition (2.3) is satisfied and when it is not. In the $(s, x)$ space, solutions converge to the equilibrium $\bar{E}$. The proofs of both main theorems will now require separate paths that we will lead in the following two sections.

\section{Proof of Theorem 1: coexistence of all competitors}

We have already proved the part of Theorem 1 pertaining to initial conditions $x_{n}(0)=0$ (Proposition 2), and will now consider $x_{n}(0)>0$. The proof of coexistence will require several steps. We will first show that the dominant competitor is uniformly persistent if Condition (2.3) is satisfied (we already knew it for the other competitors). We will then show global asymptotic stability on the manifold $v=s_{i n}$ and conclude the proof by interconnecting the $v$ and $x$ dynamics.

\subsection{Uniform persistence of all competitors.}

Proposition 3. If Condition (2.3) holds, system (2.1) is uniformly persistent.

Proof. By Lemma 3, we know that species $j$ is uniformly persistent $(j=1, \ldots, n-$ $1)$. Hence, we have to prove that the $n$-th species is uniformly persistent.

By using Remark 1, we only will consider initial conditions in $\Omega \subset \mathbb{R}_{+}^{n+1}$ and construct the functional $P: \Omega \mapsto \mathbb{R}_{+}$defined by $P(s, x)=P\left(s, x_{1}, \ldots, x_{n}\right)=x_{n}$. Notice that $P(\cdot)$ satisfies:

$$
\dot{P}=\Psi\left(s, x_{1}, \ldots, x_{n}\right) P, \quad \text { with } \quad \Psi\left(s, x_{1}, \ldots, x_{n}\right)=f_{n}(s)-D .
$$

In addition, notice that:

$$
\begin{cases}P(s, x)>0 & \text { if } \quad(s, x) \in \Omega \backslash \Gamma_{n} \\ P(s, x)=0 & \text { if } \quad(s, x) \in \Gamma_{n},\end{cases}
$$

with $\Gamma_{n}$ defined in (3.4) the face of the positive orthant corresponding to $x_{n}=0$.

Observe that $P(\cdot)$ is an average Lyapunov functional defined in the compact set $\Omega$ (see Appendix for details). Furthermore, (2.3) and statement i) from Lemma 1 imply that $\bar{s} \in\left(\lambda_{n}, \lambda_{n-1}\right)$. This fact combined with (C1) and $D=f_{n}\left(\lambda_{n}\right)$, implies

$$
\Psi(\bar{E})=f_{n}(\bar{s})-D>f_{n}\left(\lambda_{n}\right)-D=0,
$$

and the result follows by applying Proposition 4 from Appendix. 
4.2. Stability on the manifold $v=s_{i n}$. By using (3.2), system (2.1) becomes:

$$
\left\{\begin{array}{l}
\dot{v}=D s_{i n}-D v \\
\dot{x}_{j}=x_{j} f_{j}\left(v-\sum_{i=1}^{n} \gamma_{i}^{-1} x_{i}\right)+D\left[x_{j}^{0}-x_{j}\right], \quad j=1, \ldots, n-1 \\
\dot{x}_{n}=x_{n} f_{n}\left(v-\sum_{i=1}^{n} \gamma_{i}^{-1} x_{i}\right)-D x_{n}
\end{array}\right.
$$

with $x_{j}(0) \geq 0$ and $v(0)-\sum_{i=1}^{n} \gamma_{i}^{-1} x_{i}(0)=s(0) \geq 0$.

Lemma 2 states that its asymptotic behavior is described by

$$
\left\{\begin{array}{l}
\dot{x}_{j}=x_{j} f_{j}\left(s_{i n}-\sum_{i=1}^{n} \gamma_{i}^{-1} x_{i}\right)+D\left[x_{j}^{0}-x_{j}\right], \quad j=1, \ldots, n-1 \\
\dot{x}_{n}=x_{n} f_{n}\left(s_{i n}-\sum_{i=1}^{n} \gamma_{i}^{-1} x_{i}\right)-D x_{n},
\end{array}\right.
$$

with initial conditions in the compact set

$$
\mathcal{D}_{n}=\left\{\left(x_{1}, \ldots, x_{n}\right) \in \mathbb{R}_{+}^{n}: \sum_{i=1}^{n} \gamma_{i}^{-1} x_{i} \leq s_{i n}\right\} .
$$

We now show convergence to the equilibrium of the solution on the manifold $v=s_{\text {in }}$.

Lemma 5. If Condition (2.3) is satisfied, then $x^{*}=\left(x_{1}^{*}, \ldots, x_{n}^{*}\right)$ is a globally asymptotically stable equilibrium of (4.2) with respect to all initial conditions in

$$
\mathcal{K}_{n}=\left\{\left(x_{1}, \ldots, x_{n}\right) \in \mathcal{D}_{n}: x_{n}>0\right\}
$$

Proof. We will apply an adapted version of LaSalle's invariance principle (see Appendix) to system (4.2) in the compact set $\mathcal{D}_{n}$. Let $x=\left(x_{1}, \ldots, x_{n}\right)$ be a solution of (4.2). Now, we define the function $U: \mathcal{D}_{n} \mapsto \mathbb{R}$ :

$$
U(x)=\sum_{i=1}^{n} \gamma_{i}^{-1}\left(x_{i}-x_{i}^{*}\right)
$$

and the functions (with $i=1, \ldots, n$ ):

$$
\left.U_{i}^{+}\left(x_{i}\right)=\max \left\{\gamma_{i}^{-1}\left(x_{i}-x_{i}^{*}\right), 0\right)\right\} \quad \text { and } \quad U_{i}^{-}\left(x_{i}\right)=\max \left\{\gamma_{i}^{-1}\left(x_{i}^{*}-x_{i}\right), 0\right\}
$$

which allow for the definitions of

$$
U^{+}(x)=\sum_{i=1}^{n} U_{i}^{+}\left(x_{i}\right) \geq 0 \quad \text { and } \quad U^{-}(x)=\sum_{i=1}^{n} U_{i}^{-}\left(x_{i}\right) \geq 0
$$

then $U(x)=U^{+}(x)-U^{-}(x)$. Notice that by definition of $U(x)$, we have:

$$
s_{i n}-\sum_{j=1}^{n} \gamma_{j}^{-1} x_{j}=s_{i n}-\sum_{j=1}^{n} \gamma_{j}^{-1} x_{j}^{*}-U(x)=\lambda_{n}-U(x),
$$

where the last equality comes from the fact that $s_{i n}=v^{*}=s^{*}+\sum_{j=1}^{n} \gamma_{j}^{-1} x_{j}^{*}=$ $\lambda_{n}+\sum_{j=1}^{n} \gamma_{j}^{-1} x_{j}^{*}$.

Let us define the function $V: \mathcal{D}_{n} \mapsto \mathbb{R}_{+}$as follows

$$
V(x)=\max \left(U^{+}(x), U^{-}(x)\right) .
$$

$V$ is continuous, positive for all $x \in \mathcal{D}_{n} \backslash x^{*}$ and $V\left(x^{*}\right)=0$. Because of the composite structure of $V$, the stability analysis will be separated in several cases: 
Case a) $U^{+}(x)>U^{-}(x)$ : We have that $U(x)>0$ and $V(x)=U^{+}(x)$. As it was done in Lemma 4, if $x_{i}>x_{i}^{*}$, then the map $t \mapsto U_{i}^{+}\left(x_{i}(t)\right)=\gamma_{i}^{-1}\left(x_{i}-\bar{x}_{i}\right)$ is decreasing by using (4.3), for all $i \in\{1, \cdots, n-1\}$ :

$$
\dot{x}_{i}=x_{i} f_{i}\left(\lambda_{n}-U(x)\right)+D\left[x_{i}^{0}-x_{i}\right]<x_{i}\left(f_{i}\left(\lambda_{n}-U(x)\right)-f_{i}\left(\lambda_{n}\right)\right)<0,
$$

the first inequality coming from $x_{i}(t)>x_{i}^{*}=\frac{D x_{i}^{0}}{D-f_{i}\left(\lambda_{n}\right)}$ and the second one from positiveness of $U(x)$ and monotonocity of $f_{i}(\cdot)$.

Similarly, since $U(x(t))>0$, it follows from $(\mathbf{C 1})-(\mathbf{C 2})$ that, when $x_{n}>x_{n}^{*}$

$$
\dot{x}_{n}=x_{n} f_{n}\left(s_{i n}-\sum_{i=1}^{n} \gamma_{i}^{-1} x_{i}\right)-D x_{n}=x_{n}\left\{f_{n}\left(\lambda_{n}-U(x)\right)-f_{n}\left(\lambda_{n}\right)\right\}<0,
$$

the map $t \mapsto U_{n}^{+}\left(x_{n}(t)\right)$ is decreasing when $x_{n}(t)>x_{n}^{*}$.

We have then shown that, for any $i \in\{1, \cdots, n\}$, if $x_{i}(t)>x_{i}^{*}$, it contributes a decreasing term to $V(x(t))$. As in Proposition 2, when $x_{i} \leq x_{i}^{*}$, it does not contribute to the evolution of $V(x(t))$. Since $U^{+}(x)>U^{-}(x) \geq 0$, there exists some $i$ such that $x_{i}(t)>x_{i}^{*}$ so that $V(x(t))$ is decreasing along the solutions of (4.2) when $U^{+}(x)>U^{-}(x)$.

Case b) $U^{+}(x)<U^{-}(x)$ : By following the lines of the previous case, it can be proved that the map $t \mapsto U^{-}(x(t))$ is decreasing. Inequalities (4.4) and (4.5) here change sign because $U(x)<0$ and $x_{i}<x_{i}^{*}$ and $x_{n}<x_{n}^{*}$ :

$$
\begin{gathered}
\dot{x}_{i}=x_{i} f_{i}\left(\lambda_{n}-U(x)\right)+D\left[x_{i}^{0}-x_{i}\right]>x_{i}\left(f_{i}\left(\lambda_{n}-U(x)\right)-f_{i}\left(\lambda_{n}\right)\right) \geq 0 \\
\dot{x}_{n}=x_{n} f_{n}\left(s_{i n}-\sum_{i=1}^{n} \gamma_{i}^{-1} x_{i}\right)-D x_{n}=x_{n}\left\{f_{n}\left(\lambda_{n}-U(x)\right)-f_{n}\left(\lambda_{n}\right)\right\} \geq 0 .
\end{gathered}
$$

Notice that we have $\dot{x}_{n} \geq 0$ instead of $>0$ only because of the situation where $x_{n}=0$. We then have that, when $U^{-}(x)>U^{+}(x), V(x)$ is always decreasing along the solutions of (4.2), except when, simultaneously, $x_{n}=0$, no other $x_{i}$ is smaller than $x_{i}^{*}$, and $U^{-}(x)>U^{+}(x)$, that is in the set

$$
Z_{n}=\left\{x \in \mathcal{D}_{n} \mid x_{n}=0, x_{i} \geq x_{i}^{*} \quad i=1, \ldots, n-1 \text { and } U^{-}(x)>U^{+}(x)\right\} .
$$

Case c) $U^{+}(x)=U^{-}(x)$ : By (4.3) combined with $U(x)=0$, we can deduce that the solution $x(t)$ satisfies

$$
\dot{x}_{i}(t)=x_{i}(t) f_{i}\left(\lambda_{n}\right)+D\left[x_{i}^{0}-x_{i}(t)\right], \quad i=1, \ldots, n-1,
$$

which is negative when $x_{i}>x_{i}^{*}$ as it was shown in (4.4) and positive when $x_{i}<x_{i}^{*}$ so that, in these situations, $x_{i}$ contributes a decreasing term to $V(x)$.

In the case $i=n$, the solution $x(t)$ satisfies

$$
\dot{x}_{n}(t)=x_{n}(t) f_{n}\left(\lambda_{n}\right)-D x_{n}(t)=0,
$$

and this case is different from the others. If $x_{n}<x_{n}^{*}$ and all other $x_{i} \geq x_{i}^{*}$ (with $U(x)=0), x_{n}$ is the only one to contribute to $U^{-}(x)$ for which we will not be able to show that it is decreasing.

In that line of thought, let us define the sets:

$$
\begin{gathered}
\Sigma=\left\{\left(x_{1}, \ldots, x_{n}\right) \in \mathcal{D}_{n}: U(x)=0\right\} . \\
\Sigma_{n}^{-}=\left\{\left(x_{1}, \ldots, x_{n}\right) \in \mathcal{D}_{n}: U(x)=0, \quad \text { and } \quad x_{i} \geq x_{i}^{*} \quad i=1, \ldots, n-1\right\} . \\
\Sigma_{n}^{+}=\left\{\left(x_{1}, \ldots, x_{n}\right) \in \mathcal{D}_{n}: U(x)=0, \quad \text { and } \quad x_{i} \leq x_{i}^{*} \quad i=1, \ldots, n-1\right\} .
\end{gathered}
$$


Since $U(x)=0$ and $x_{i} \geq x_{i}^{*}$ for $i=1, \ldots, n-1$ in $\Sigma_{n}^{-}$, we obviously have $x_{n} \leq x_{n}^{*}$ in $\Sigma_{n}^{-}\left(\right.$resp. $x_{n} \geq x_{n}^{*}$ in $\left.\Sigma_{n}^{+}\right)$.

These sets are the only critical cases when $U^{+}(x)=U^{-}(x)$ since, if $x(t) \in$ $\Sigma \backslash\left(\Sigma_{n}^{+} \cup \Sigma_{n}^{-}\right)$, there exists two indices $j$ and $k$ (both $\left.\leq n-1\right)$ such that $x_{j}>x_{j}^{*}$ and $x_{k}<x_{k}^{*}$. Following the lines of Cases a) and b), we have that $U^{+}(x(t))$ and $U^{-}(x(t))$ are decreasing, which implies that $t \mapsto V(x(t))$ is decreasing.

If $x(t) \in \Sigma_{n}^{-} \backslash\left\{x^{*}\right\}$, there exists $i \in\{1, \ldots, n-1\}$ such that $x_{i}(t)>x_{i}^{*}$ (and we have that $x_{n}(t)<x_{n}^{*}$ ). Indeed, otherwise we have that $x_{i}(t)=x_{i}^{*}$ for all $i=1, \ldots, n-1$ which, combined with $U(x(t))=0$, implies that $x_{n}(t)=x_{n}^{*}$ obtaining a contradiction. In consequence, for the $i$ such that $x_{i}(t)>x_{i}^{*}$, it follows that $\dot{x}_{i}(t)<0$ and the map

$$
t \rightarrow U^{+}(x(t))
$$

is decreasing, whereas the map

$$
t \rightarrow U^{-}(x(t))
$$

which is equivalent to $t \rightarrow \gamma_{n}^{-1}\left(x_{n}^{*}-x_{n}(t)\right)$ is constant. A similar reasoning can be held for $x(t) \in \Sigma_{n}^{+} \backslash\left\{x^{*}\right\}$.

Application of LaSalle's principle: In consequence, the map $t \mapsto V(x(t))$ is decreasing for all values of $x \in \mathcal{D}_{n}$ except, potentially, for either $x \in\left(\Sigma_{n}^{+} \cup \Sigma_{n}^{-}\right)$, which contains $x^{*}$ or $x \in Z_{n}$. By LaSalle's invariance principle (see Appendix), it follows that every solution of (4.2) is convergent to the largest invariant set $M \subseteq\left(\Sigma_{n}^{+} \cup \Sigma_{n}^{-} \cup Z_{n}\right)$.

Now, we will verify that $M \subseteq\left\{x^{*}\right\} \cup \operatorname{cl}\left(Z_{n}\right)$ : let us consider some $x \in \Sigma_{n}^{-} \backslash\left\{x^{*}\right\}$. We have seen that, for that $x$, the map $t \rightarrow U^{+}(x(t))$ is decreasing while $t \rightarrow$ $U^{-}(x(t))$ stays constant. As a consequence, the map $t \rightarrow U(x)=U^{+}(x(t))-$ $U^{-}(x(t))$ is decreasing for that $x$. We then have that $U(x(t))$ becomes negative so that the solution leaves $\left(\Sigma_{n}^{+} \cup \Sigma_{n}^{-}\right)$. This means that, in this case, $x(t)$ leaves $\left(\Sigma_{n}^{+} \cup\right.$ $\left.\Sigma_{n}^{-} \cup Z_{n}\right)$ so that the chosen $x$ can therefore not belong to $M$, except potentially if the initial condition belonged to the closure of $Z_{n}$; in this latter situation, and since $\left(\Sigma_{n}^{+} \cup \Sigma_{n}^{-}\right) \cap Z_{n}=\emptyset$, the solution could instantaneously enter $Z_{n}$.

A similar reasoning follows for any point $x \in \Sigma_{n}^{+} \backslash\left\{x^{*}\right\}$ : the largest invariant subset of $\left(\Sigma_{n}^{+} \cup \Sigma_{n}^{-} \cup Z_{n}\right)$ is contained in $\left\{x^{*}\right\} \cup \operatorname{cl}\left(Z_{n}\right)$ and all solutions of (4.2) converge either to $x^{*}$ or to $x_{n}=0$.

The proof finish by noting that, as we only consider initial conditions in $\mathcal{K}_{n}$ and $x_{n}$ is persistent (Proposition 3), any solution with initial condition in $\mathcal{K}_{n}$ converges to $x^{*}$, which is asymptotically stable because $V$ is locally a Lyapunov function.

Remark 4. We have just shown asymptotic stability of $s_{\text {in }}$ for the $\dot{v}$ dynamics (3.3) in Lemma 2 and of $x^{*}$ for the $\dot{x}$ dynamics (4.2) in Lemma 5. Hence, by Theorem 3.2 from [40] it follows that $\left(s_{i n}, x_{1}^{*}, \ldots, x_{n}^{*}\right)$ is a LAS equilibrium of (4.1) when Condition (2.3) is satisfied.

4.3. Stability for the full system. In this subsection, no new theorem statement is needed, we simply conclude the proof of Theorem 1.

We will, however, prove an equivalent statement: the global stability of the equilibrium $E_{v}^{*}=\left(s_{i n}, x_{1}^{*}, \ldots, x_{n}^{*}\right)$ of $(4.1)$ : let $\left(v_{0}, x_{0}\right) \in \mathbb{R}_{+} \times \mathbb{R}_{+}^{n}$ an initial condition of (4.1) with $x_{0 n}>0$ and $v_{0}-\sum_{i=1}^{n} \gamma_{i}^{-1} x_{0 i} \geq 0$. Denote by $\phi_{t}\left(v_{0}, x_{0}\right)$ its associated 
semiflow, the $\omega$-limit set of $\left(v_{0}, x_{0}\right)$ is defined by:

$$
\omega\left(v_{0}, x_{0}\right)=\left\{(\tilde{v}, \tilde{x}) \in \mathbb{R}_{+} \times \mathbb{R}_{+}^{n}: \exists t_{n} \rightarrow+\infty \quad \lim _{n \rightarrow+\infty} \phi_{t_{n}}\left(v_{0}, x_{0}\right)=(\tilde{v}, \tilde{x})\right\} .
$$

Given any $(\tilde{v}, \tilde{x}) \in \omega\left(v_{0}, x_{0}\right)$, Lemma 2 implies that $\omega\left(v_{0}, x_{0}\right)$ is non empty, and $\tilde{v}=s_{i n}$; by using invariance of $\omega\left(v_{0}, x_{0}\right)$ it follows that $\phi_{t}\left(s_{i n}, \tilde{x}\right) \in \omega\left(v_{0}, x_{0}\right)$ for any $t \geq 0$. We point out that the set

$$
\tilde{\Gamma}_{n}=\left\{\left(v, x_{1}, \ldots, x_{n}\right) \in \mathbb{R}_{+}^{n+1}: x_{n}=0 \text { and } v-\sum_{i=1}^{n} \gamma_{i}^{-1} x_{i} \geq 0\right\}
$$

is also invariant under the semiflow. Nevertheless, the persistence of the species (see Proposition 3) implies that $\tilde{\Gamma}_{n}$ cannot be attractive. Hence, we have that:

$$
\omega\left(v_{0}, x_{0}\right) \cap \tilde{\Gamma}_{n}=\emptyset .
$$

In addition, we have that the $x$ component of $\phi_{t}\left(s_{i n}, \tilde{x}\right) \in \omega\left(v_{0}, x_{0}\right)$ is a solution of (4.2) with initial condition $x(0)=\tilde{x}$. By letting $t \rightarrow+\infty$, it follows by Lemma 5 that $E_{v}^{*} \in \omega\left(v_{0}, x_{0}\right)$ and in consequence, there exists a divergent sequence $\left\{t_{k}\right\}_{k}$ such that $\phi_{t_{k}}\left(v_{0}, x_{0}\right)$ converges toward $E_{v}^{*}$.

Finally, by Remark 4 we know that $E_{v}^{*}$ is also LAS stable, which implies that in a finite time $t_{k}$, the orbit $\phi_{t_{k}}\left(v_{0}, x_{0}\right)$ enters the basin of attraction of $E_{v}^{*}$ and the Theorem follows.

\section{Proof of Theorem 2: extinction of the Dominant Competitor}

We now want to show that we have global asymptotic stability of $\bar{E}$. We therefore will follow the path of Lemma 5 in the following lemma

Lemma 6. If Condition (2.3) is strictly not satisfied, then $\bar{x}=\left(\bar{x}_{1}, \ldots, \bar{x}_{n-1}, 0\right)$ is a GAS equilibrium of (4.2) with respect to all initial conditions in $\mathcal{D}_{n}$.

Proof. The proof is identical to that of Lemma 5 through the construction of a function $U(x)=\sum_{i=1}^{n} \gamma_{i}^{-1}\left(x_{i}-\bar{x}_{i}\right)$ where we define $\bar{x}_{n}=0$. The definitions of the $U_{i}^{ \pm}(x), U^{ \pm}(x)$ and $V(x)$ functions follow and the analysis is unchanged compared to that of the previous proof. There are however two small differences:

- The set $Z_{n}$ defined in (4.6) is empty because $x_{n}^{*}$ is here replaced by 0 and the last two inequalities in the definition of $Z_{n}$ are not compatible.

- $\Sigma_{n}^{-}$reduces to the equilibrium $\bar{x}$ and does not come into play in the LaSalle part of the proof.

These two differences have no fundamental implication in the flow of the proof; they only simplify it. All solutions then converge to $\bar{x}$, which is asymptotically stable because $V(x)$ locally is a Lyapunov function.

We will now prove an equivalent statement to Theorem 2: the global stability of the unique non-negative equilibrium (uniqueness is ensured by Lemma 1) $\bar{E}_{v}=$ $\left(s_{i n}, \bar{x}_{1}, \ldots, \bar{x}_{n-1}, 0\right)$ of (4.1): let $\left(v_{0}, x_{0}\right) \in \mathbb{R}_{+} \times \mathbb{R}_{+}^{n}$ an initial condition of (4.1) with $v_{0}-\sum_{i=1}^{n} \gamma_{i}^{-1} x_{0 i} \geq 0$. Denote by $\phi_{t}\left(v_{0}, x_{0}\right)$ the semiflow defined by (4.1). The $\omega$-limit set of $\left(v_{0}, x_{0}\right)$ is defined by:

$$
\omega\left(v_{0}, x_{0}\right)=\left\{(\tilde{v}, \tilde{x}) \in \mathbb{R}_{+} \times \mathbb{R}_{+}^{n}: \exists t_{n} \rightarrow+\infty \quad \lim _{n \rightarrow+\infty} \phi_{t_{n}}\left(v_{0}, x_{0}\right)=(\tilde{v}, \tilde{x})\right\} .
$$


Given any $(\tilde{v}, \tilde{x}) \in \omega\left(v_{0}, x_{0}\right)$, Lemma 2 implies that $\omega\left(v_{0}, x_{0}\right) \neq \emptyset$ and $\tilde{v}=s_{\text {in }}$; by invariance of $\omega\left(v_{0}, x_{0}\right)$ it follows that $\phi_{t}\left(s_{i n}, \tilde{x}\right) \in \omega\left(v_{0}, x_{0}\right)$ for any $t \geq 0$.

In addition, we have that the $x$ component of $\phi_{t}\left(s_{i n}, \tilde{x}\right) \in \omega\left(v_{0}, x_{0}\right) \in \Gamma_{n}$ is a solution of (3.7) with initial condition $x(0)=\tilde{x}$. By letting $t \rightarrow+\infty$, it follows by Lemma 4 that $\bar{E}_{v} \in \omega\left(v_{0}, x_{0}\right)$ and in consequence, there exists a divergent sequence $\left\{t_{k}\right\}_{k}$ such that $\phi_{t_{k}}\left(v_{0}, x_{0}\right)$ converges toward $\bar{E}_{v}$.

Finally, as before, we know that $\bar{E}_{v}$ is also locally asymptotically stable through the use of Lemma 2 and Lemma 6 . This implies that, after some finite time $t_{k}$, the orbit $\phi_{t_{k}}$ enters the basin of attraction of $\bar{E}_{v}$ and the Theorem follows.

\section{An ARBitrary NUMBER OF SPECIES IN THE INPUT}

Up to now, we have focused our attention on a very particular situation: the case where the $n-1$ inferior species, and only them, are fed into the system. In this section, we will consider three extensions of that result: the cases where

- all species are fed into the system;

- $n-1$ species, including the dominant one, are fed into the system;

- $n-k$ species (with $k>1$ ) are fed into the system.

6.1. All species are in the input flow. In fact, no new theorem needs to be proved here. The result has already been given in Proposition 2. When considering the system in absence of the dominant species, we have just studied the system where all species are fed into the system. To summarize Proposition 2, there is a single equilibrium which is globally asymptotically stable in $\mathbb{R}_{+}^{n}$. Persistence was obviously trivial, as we had done in Lemma 3; the result of Proposition 2 only adds the global asymptotic stability.

6.2. Only one inferior species is not in the input flow. This case can easily be handled through an equilibria study. If species $m<n$ is the only one not in the input flow, its dynamics are

$$
\dot{x}_{m}=x_{m} f_{m}(s)-D x_{m}
$$

which can be at equilibrium either if $x_{m}=0$ or $s=\lambda_{m}$. In the former case, a single equilibrium in the form $\bar{E}_{m}=\left(\bar{s}, \bar{x}_{1}, \cdots, \bar{x}_{m-1}, 0, \bar{x}_{m+1}, \cdots, \bar{x}_{n}\right)$ arises in exactly the same manner as in Lemma 1 . When considering $s=\lambda_{m}$, it suffices to consider the $\dot{x}_{n}$ dynamics

$$
\dot{x}_{n}=x_{n} f_{n}(s)-D x_{n}+D x_{n}^{0}
$$

At $s=\lambda_{m}>\lambda_{n}$, the sum of the first two terms is already non negative, so that $\dot{x}_{n}>0$. Species $x_{n}$ can therefore not be at equilibrium with $s=\lambda_{m}$. There is therefore a single equilibrium in the present case, the one where $x_{m}$ is washed-out. It is not surprising since $x_{m}$ was already not able to compete with $x_{n}$ when $x_{n}$ was not fed into the system; there is no way $x_{m}$ could survive with this additional advantage to $x_{n}$.

We then have the following theorem

Theorem 3. Assume that (C1)-(C3) are satisfied, then system

$$
\left\{\begin{aligned}
\dot{s} & =D\left[s^{0}-s\right]-\sum_{i=1}^{n} \gamma_{i}^{-1} f_{i}(s) x_{i}, & & \\
\dot{x}_{j} & =x_{j} f_{j}(s)+D\left[x_{j}^{0}-x_{j}\right], & & j=1, \ldots, m-1, m+1, \ldots, n \\
\dot{x}_{m} & =x_{m} f_{m}(s)-D x_{m}, & & m \neq n,
\end{aligned}\right.
$$


with $s(0), x_{j}(0), x_{m}(0) \geq 0$, has a GAS single equilibrium $\bar{E}_{m} \in \partial \mathbb{R}_{+}^{n+1}$ defined by

$$
\bar{E}_{m}=\left(\bar{s}, \bar{x}_{1}, \cdots, \bar{x}_{m-1}, 0, \bar{x}_{m+1}, \cdots, \bar{x}_{n}\right) \quad \text { with } \quad \bar{x}_{j}=\frac{D x_{j}^{0}}{D-f_{j}(\bar{s})} \quad \text { for } \quad j \neq m
$$

and $\bar{s} \in\left(0, \lambda_{n}\right)$ is the unique fixed point of the map $\mathcal{G}_{m}:\left[0, \lambda_{n}\right) \mapsto \mathbb{R}$ :

$$
\mathcal{G}_{m}(s)=s_{i n}-\sum_{j=1, j \neq m}^{n} \frac{\gamma_{j}^{-1} D x_{j}^{0}}{D-f_{j}(s)} .
$$

No additional proof needs to be given since it follows exactly in the footsteps of the proof of Theorem 2 .

6.3. Only $n-k$ species are in the input flow. We have already proved the result for $k=0$ and $k=1$ and the result is classical for $k=n$, where there is no biomass input. We are only left with the proof in the case where $2 \leq k \leq n-1$. We can write the system that we will study as follows:

$$
\left\{\begin{aligned}
\dot{s} & =D\left[s^{0}-s\right]-\sum_{i=1}^{n} \gamma_{i}^{-1} f_{i}(s) x_{i}, & & \\
\dot{x}_{j} & =x_{j} f_{j}(s)+D\left[x_{j}^{0}-x_{j}\right], & & j \in \mathcal{F} \\
\dot{x}_{j} & =x_{j} f_{j}(s)-D x_{j}, & & j \notin \mathcal{F}
\end{aligned}\right.
$$

with $s(0), x_{j}(0) \geq 0$ and where $\mathcal{F}$ is the set of indices of the species that are fed into the system (the cardinal of $\mathcal{F}$ is $n-k$ with $0 \leq k \leq n$ ). We can then define the equilibria as follows.

Let $L$ be the largest index contained in $\mathcal{F}$; if $L<n$ (or, equivalently, $n \notin \mathcal{F}$ ) then, for any $i>L, x_{i}$ is not fed into the system and we potentially have an equilibrium $E^{* i}=\left(s^{* i}, x^{* i}\right)$ in the form

$$
s^{* i}=\lambda_{i} \text { and } x^{* i}=\left\{\begin{array}{rlrl}
x_{j}^{* i} & =\frac{D x_{j}^{0}}{D-f_{j}\left(\lambda_{i}\right)} & \text { for } j \in \mathcal{F} \\
x_{i}^{* i} & =\gamma_{i}\left\{s_{i n}-\lambda_{i}-\sum_{j \in \mathcal{F}} \gamma_{j}^{-1} x_{j}^{* i}\right\} & \\
x_{j}^{* i} & =0 & & \text { otherwise }
\end{array}\right.
$$

Note that, since $i>j$ for all $j \in \mathcal{F}$, all $x_{j}^{* i}$ with $j \in \mathcal{F}$ are positive. The only question is whether $x_{i}^{* i}$ is positive, which requires that

$$
\sum_{j \in \mathcal{F}} \frac{\gamma_{j}^{-1} D x_{j}^{0}}{D-f_{j}\left(\lambda_{i}\right)}<s_{i n}-\lambda_{i}
$$

Note that the left-hand-side of this inequality is an increasing function of $\lambda_{i}$ while the right-hand-side is a decreasing function of $\lambda_{i}$. We can then conclude that, provided $L<n$, the set of equilibria of the type $E^{* i}$ is either empty or contains at least $E^{* n}$. The condition of existence of at least one equilibrium of this kind is therefore the condition of existence of $E^{* n}$.

\section{$E^{* n}$ Existence Condition:}

$$
n \notin \mathcal{F} \text { and } \sum_{j \in \mathcal{F}} \frac{\gamma_{j}^{-1} D x_{j}^{0}}{D-f_{j}\left(\lambda_{n}\right)}<s_{i n}-\lambda_{n}
$$


As we have seen in the previous subsection, we cannot build an equilibrium having $x_{i}>0$ for $i \notin \mathcal{F}$ and $i<L$ because we would have $\dot{x}_{L}>0$. The only remaining equilibrium is therefore $\bar{E}=(\bar{s}, \bar{x})$ built as in Lemma 1

$$
\bar{x}= \begin{cases}\bar{x}_{j}=\frac{D x_{j}^{0}}{D-f_{j}(\bar{s})} & \text { for } j \in \mathcal{F} \\ \bar{x}_{j}=0 & \text { for } j \notin \mathcal{F}\end{cases}
$$

with $\bar{s}$ the only fixed point of

$$
\mathcal{G}_{\mathcal{F}}(s)=s_{i n}-\sum_{j \in \mathcal{F}} \frac{\gamma_{j}^{-1} D x_{j}^{0}}{D-f_{j}(s)} .
$$

Note that, as was done previously, $\mathcal{G}_{\mathcal{F}}\left(\lambda_{n}\right)=s_{i n}-\sum_{j \in \mathcal{F}} \frac{\gamma_{j}^{-1} D x_{j}^{0}}{D-f_{j}\left(\lambda_{n}\right)}<\lambda_{n}$ if the $E^{* n}$ Existence Condition (6.3) is not satisfied, so that $\bar{s}<\lambda_{n}$ in that case (and vice-versa if (6.3) is satisfied).

We then have the following result:

Theorem 4. Assume that (C1)-(C3) are satisfied. If the $E^{* n}$ Existence Condition (6.3) is satisfied then all solutions of (6.2) with initial condition in $\mathbb{R}_{+}^{n+1}$ satisfying $x_{n}(0)>0$ converge to the stable equilibrium $E^{* n}$. If the $E^{* n}$ Existence Condition is strictly not satisfied, the equilibrium $\bar{E}$ is $G A S$ in $\mathbb{R}_{+}^{n+1}$.

Proof. We will use a proof by induction. Suppose that the result is valid for $n-1$ and prove it for $n$ (the result is obviously valid for $\mathrm{n}=1$ ).

Assuming that Condition (6.3) is satisfied, we have equilibrium $\bar{E}$ and $p$ equilibria in the form $E^{* i}$ (with $0<p \leq n-L$ where $L$ is the largest index of $\mathcal{F}$ ): $E^{* n}, E^{* n-1}, \cdots, E^{* n-p+1}$. Of these equilibria, only $E^{* n}$ has $x_{n}>0$. We will first show persistence of $x_{n}$, which will prevent convergence to the face where $x_{n}=0$. For that, we will do as in Proposition 3: we only will consider initial conditions in the compact set $\Omega \subset \mathbb{R}_{+}^{n+1}$ defined after Lemma 2 and construct the functional $P: \Omega \mapsto \mathbb{R}_{+}$defined by $P(s, x)=x_{n}$ such that

$$
\dot{P}=\Psi\left(s, x_{1}, \ldots, x_{n}\right) P, \quad \text { with } \quad \Psi\left(s, x_{1}, \ldots, x_{n}\right)=f_{n}(s)-D .
$$

The induction hypothesis indicates that all initial conditions such that $x_{n}(0)=0$ lead to solutions converging to one of the equilibria within this face, that is $\bar{E}$ or $E^{* n-1}, \cdots, E^{* n-p+1}$. Also we have

$$
\Psi(\bar{E})=f_{n}(\bar{s})-D>0 \quad \text { and } \quad \Psi\left(E^{* i}\right)=f_{n}\left(\lambda_{i}\right)-D>0
$$

Proposition 4 then implies that the species $x_{n}$ is uniformly persistent, so that if solutions converge, they need to do so towards $E^{* n}$. Since the persistence of all species that are fed into the chemostat is trivial, the persistence of all species that appear in $E^{* n}$ is shown.

The continuation of the proof is then identical to that of Lemma 5 through the study of model (6.2) on the manifold where $s+\sum_{j=1}^{n} \gamma_{j}^{-1} x_{j}=s_{i n}$, and is not given for the sake of brevity.

This proofs rounds up the results of this paper. We have shown that the presence of biomass in the inputs results in a globally asymptotically stable equilibrium, independently of the situation. 


\section{Numerical EXAmple}

Let us consider the system

$$
\left\{\begin{array}{l}
\dot{s}=D\left[s^{0}-s\right]-\sum_{i=1}^{3} \gamma_{i}^{-1} f_{1}(s) x_{i} \\
\dot{x}_{1}=x_{1} f_{1}(s)+D x_{1}^{0}-D x_{1} \\
\dot{x}_{2}=x_{2} f_{2}(s)+D x_{2}^{0}-D x_{2} \\
\dot{x}_{3}=x_{3} f_{3}(s)-D x_{3}
\end{array}\right.
$$

with parameters ( $m g, h$ and $l$ denote miligrams, hour and liters respectively):

$$
D=0.2[l / h], \quad s_{i n}=2[m g / l], \quad \gamma_{1}=10, \quad \gamma_{2}=2 \quad \text { and } \quad \gamma_{3}=0.5 .
$$

The functions $f_{i}$ are of type Michaelis-Menten [38]:

$$
f_{1}(s)=\frac{1.6[1 / h] s}{0.2\left[\frac{\mathrm{mg}}{\mathrm{l}}\right]+s}, \quad f_{2}(s)=\frac{1.4[1 / \mathrm{h}] s}{0.03\left[\frac{\mathrm{mg}}{\mathrm{l}}\right]+s} \quad \text { and } \quad f_{3}(s)=\frac{0.9[1 / \mathrm{h}] s}{0.002\left[\frac{\mathrm{mg}}{\mathrm{l}}\right]+s},
$$

which realistic parameters (see e.g. [2],[39]), leading to the values:

$$
\lambda_{1}=0.028571, \quad \lambda_{2}=0.005 \text { and } \lambda_{3}=0.00057143
$$

and it follows that condition (C3) is satisfied.

If we consider the inputs

$$
s^{0}=1.4, \quad x_{1}^{0}=1 \quad \text { and } \quad x_{2}^{0}=1,
$$

it can be checked that:

$$
1.9994=s_{i n}-\lambda_{2}>\frac{\gamma_{1}^{-1} D x_{1}^{0}}{D-f_{1}\left(\lambda_{3}\right)}+\frac{\gamma_{2}^{-1} D x_{2}^{0}}{D-f_{2}\left(\lambda_{3}\right)}=0.6778
$$

and condition (2.3) is verified. Hence, by Theorem 1 there exists a globally attractive positive equilibrium $\left(x_{1}^{*}, x_{2}^{*}, x_{3}^{*}\right)=(1.0235,1.1507,0.66)$. The figures 2 and 3 shows a numerical example with initial conditions $(0.001,0.1,0.1,0.1)$.
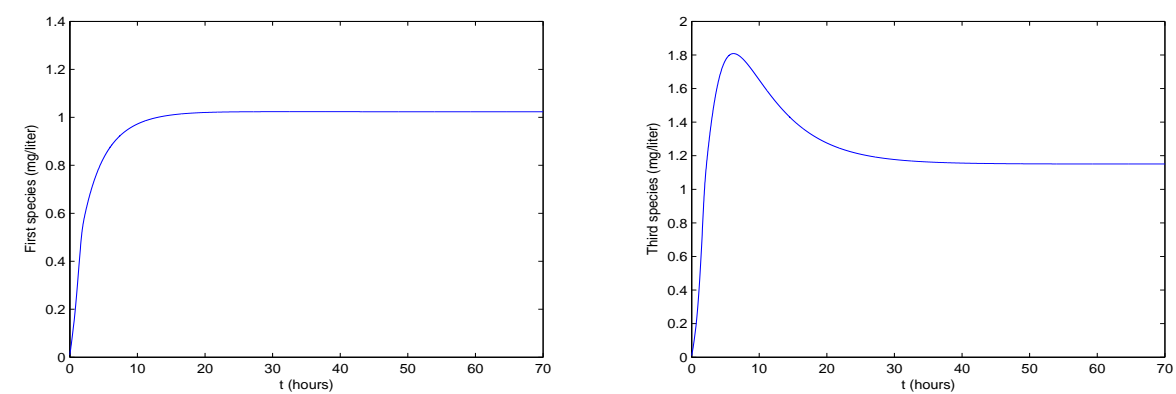

Figure 2. Results for (7.1) with parameters (7.2), functions (7.3) and inputs (7.4): first species (left), second species (right).

Now, let us consider the inputs

$$
s^{0}=0.005, \quad x_{1}^{0}=3.7 \quad \text { and } \quad x_{2}^{0}=3.25,
$$

it can be checked that condition (2.3) is not verified since

$$
1.9994=s_{i n}-\lambda_{2}<\frac{\gamma_{1}^{-1} D x_{1}^{0}}{D-f_{1}\left(\lambda_{3}\right)}+\frac{\gamma_{2}^{-1} D x_{2}^{0}}{D-f_{2}\left(\lambda_{3}\right)}=2.0174 .
$$




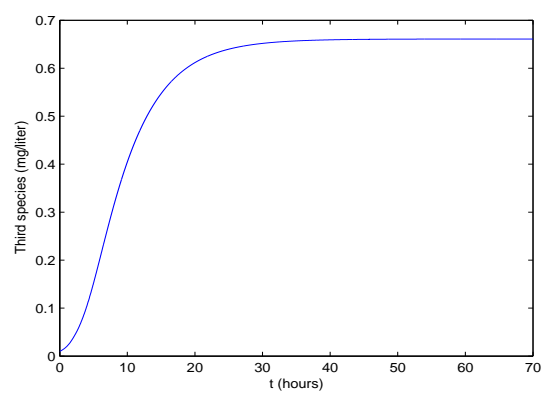

FiguRE 3. Thirds species of (7.1) with parameters (7.2), functions (7.3) and inputs (7.4).

Theorem 2 implies the existence of a globally stable equilibrium $\left(\bar{s}, \bar{x}_{1}, \bar{x}_{2}, 0\right)$. Figures 4 and 5 shows a numerical example with initial conditions $(0.001,0.1,0.1,0.1)$.
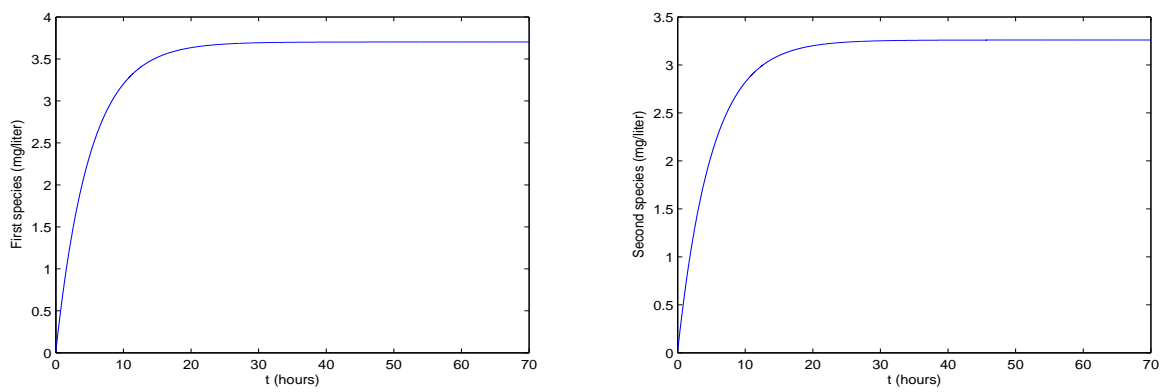

Figure 4. Results for (7.1) with parameters (7.2), functions (7.3) and inputs (7.5): first species (left), second species (right).

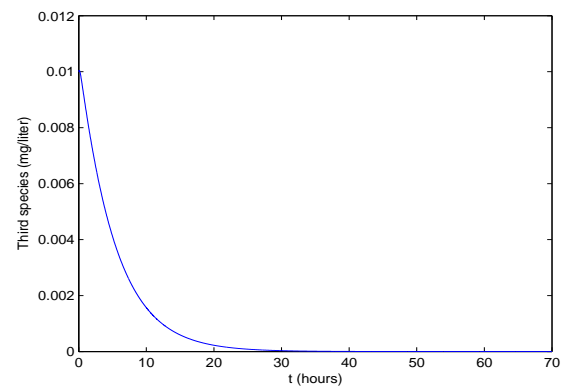

Figure 5. Third species in (7.1) with parameters (7.2), functions (7.3) and inputs (7.5).

We check that the dominant species goes to extinction, because of the larger values of inputs of other species. It can be seen as a way of controlling the dominant species by acting on the inputs of the inferior ones. 


\section{Discussion}

We considered a chemostat model with $n$ species competing for a single limiting substrate. Two questions were considered: how can we ensure coexistence of all species through the presence of biomass in the input and what are the consequences of the presence of this biomass on the stability of the system.

We answered the first question by showing that it was necessary to have at least the presence of the $n-1$ inferior species in the inputs, but that this was no guarantee for complete coexistence. Indeed, if the densities in the input are too high, the inferior biomasses consume too much substrate for the superior biomass to survive. This is the meaning of the Coexistence Condition (2.3). The strength of our result is that the latter condition is a necessary and sufficient condition for coexistence: if it is verified, all species coexist, if not, the superior species is washed-out of the chemostat which settles at a substrate level which is lower than the break-even concentration of all species, the inferior species being maintained only through the input and a little growth.

An alternative interpretation could be consider (2.1) as a perturbation of the classical model $(1.1)$ by a vector $\Delta=D\left(x_{1}^{0}, \ldots, x_{n-1}^{0}, 0\right)^{T} \in \mathbb{R}_{+}^{n}$. Theorems $1-2$ then show that small perturbations promote biodiversity while larger ones might not be as beneficial. This is a classical result in theoretical ecology [4].

Alternatively, our result offers a way of practically leading the dominant species to extinction in a community without input of this species: it suffices, starting from a coexistence situation with some inputs, to increase slowly the inputs of the inferior species; above some threshold given by our condition (2.3), the dominant species will be asymptotically eliminated, which in practice leads to effective suppression in finite time. The return to the initial inputs is then possible, the dominant species being now absent from the chemostat.

Finally, in all situations of Theorems 1-4, the stable equilibrium was the one corresponding to the smallest value of the substrate: this was obviously valid when there was a single equilibrium but we have also seen that, if several equilibria were present, only the one having $s=\lambda_{n}$ (smaller than all other $\lambda_{i}$ and smaller than $\bar{s}$ in that case) was stable. This is in line with the classical competitive exclusion and other results concerning competition of Droop species [18] or competition between a mix of generalized Monod, Droop and Contois species [25]. The parallel with the latter paper extends beyond this observation since at most one unperturbed generalized Monod species can survive in the chemostat with all species that are fed into the system (current paper) and with some of the Contois species [25].

\section{Appendix}

\section{Average Lyapunov functions and uniform persistence.}

Definition 2 (See [16]). Let $\phi_{t}$ be a semiflow defined in a compact metric space $(X, d)$ and let $\Gamma$ a closed and invariant subset of $X$. An application $P: X \mapsto \mathbb{R}_{+}$is an average Lyapunov function if the following properties are satisfied:

$$
\begin{gathered}
P(u)>0 \text { for } u \in X \backslash \Gamma \text { and } P(u)=0 \text { for } u \in \Gamma . \\
\dot{P}=\Psi(u) P \text { with } \Psi: X \mapsto \mathbb{R} \text { continuous. }
\end{gathered}
$$


Proposition 4 (See [16]). Let $P$ an Average Lyapunov function and let

$$
\Lambda=\left\{r_{i} \in \Gamma: \phi_{t}\left(r_{i}\right)=r_{i} \quad \text { for any } t \in \mathbb{R}\right\} .
$$

If $\phi_{t}(u) \rightarrow r_{i}$ when $t \rightarrow+\infty$ and $\Psi\left(r_{i}\right)>0$ for any $u \in \Gamma$ and $r_{i} \in \Lambda$, then $\Gamma$ is a repeller, that means $\omega(x) \notin \Gamma$ for any $x \in X \backslash \Gamma$.

LaSalle invariance principle. Consider the ODE system:

$$
\dot{x}=f(x)
$$

where $f: \mathcal{D} \subset \mathbb{R}^{n} \mapsto \mathbb{R}^{n}$ is such that its associated semiflow is well defined.

The LaSalle invariance principle is employed for studying the stability properties of (8.3) and its extension to the piecewise differentiable case has been suggested in several works (see e.g., [3]). We present a result for the sake of completeness:

Proposition 5 (Invariance Principle). Let $\mathcal{K} \subset \mathcal{D}$ be a compact set that is positively invariant under the semiflow defined by (8.3). Let $V: \mathcal{D} \subseteq \mathcal{D} \mapsto \mathbb{R}$ be a continuous function such that $t \rightarrow V\left(\phi_{t}\left(q_{0}\right)\right)$ is non-increasing when $q_{0} \in \mathcal{D}$. Let $E$ be the set of all the points in $\mathcal{K}$ such that $V\left(\phi_{t}(\cdot)\right)$ is constant. Let $M$ be the largest invariant set in $E$. Then, every solution starting in $\mathcal{K}$ approaches $M$ as $t \rightarrow+\infty$.

Proof. Note that $V(\cdot)$ is bounded on $\mathcal{K}$. We can deduce that the map $t \mapsto V\left(\phi_{t}\left(q_{0}\right)\right)$ is non-increasing and lowerly bounded and the following limit is well defined:

$$
\lim _{t \rightarrow+\infty} V\left(\phi_{t}\left(q_{0}\right)\right)=a \text {. }
$$

In addition, for any $p \in \omega\left(q_{0}\right)$, there exists a divergent sequence $\left\{t_{n}\right\}$ such that $\phi_{t_{n}}\left(q_{0}\right) \rightarrow p$ when $n \rightarrow+\infty$. By continuity of $V(\cdot)$ and using (8.4), it follows that

$$
\lim _{n \rightarrow+\infty} V\left(\phi_{t_{n}}\left(q_{0}\right)\right)=V(p)=a
$$

and we can conclude that $V(\cdot)$ is constant in the $\omega$-limit set $\omega\left(q_{0}\right)$. Thus,

$$
\omega\left(q_{0}\right) \subset M \subset E \subset \mathcal{K} \text {. }
$$

The result follows since any positive orbit approaches its $\omega$-limit at $t=+\infty$.

Acknowledgements: The authors acknowledge contributions of G. Bastin (Center for Systems Engineering and Applied Mechanics, Louvain-la-Neuve, Belgium) for

discussions of first versions of this work. The first author acknowledges the support of INRIA BIOCORE-INRIA for a travel grant.

\section{REFERENCES}

[1] J. Arino, S. Pilyugin, G.S.K. Wolkowicz, Considerations on yield, nutrient uptake, cellular growth, and competition in chemostat models. Canad.Appl.Math.Quart. 11 (2003) 107-142.

[2] O. Bernard, G. Malara, A. Sciandra, The effects of a controlled fluctuating nutrient environment on continous cultures of phytoplankton monitored by computers. J.Exp.Mar.Biol.Ecol. 197 (1996) 263-278.

[3] S. Chareyron, Stabilité des Systèmes dynamiques Non-Réguliers, Application aux Robots Marcheurs. PhD Thesis, Institut National Polytechnique de Grenoble, Grenoble, 2005.

[4] J.H. Connel, Diversity in tropical rain forests and coral reefs. Science. 199 (1978) 1302-1310.

[5] P. De Leenheer, H.L. Smith, Feedback control for chemostat models. J.Math.Biol. 46 (2003) 48-70.

[6] P. De Leenheer, S. Pilyugin, Feedback-mediated oscillatory coexistence in the chemostat. in: C. Commault, N. Marchand (Eds.) Proceedings of the second Multidisciplinary International Symposium on Positive Systems: Theory and Applications (POSTA 06), Lecture Notes in Control and Information Sciences 341, Springer-Verlag, pp. 97-104, 2006. 
[7] P. De Leenheer, D. Angeli, E.D. Sontag, Crowding effects promote coexistence in the chemostat. J.Math.Anal.Appl. 319 (2006), 48-60.

[8] S. Dikshitulu, B.C. Baltzis, G.A. Lewandowski, S. Pavlou, Competition between two microbial populations in a sequencing fed-batch reactor: Theory, experimental verification, and implications for waste treatment applications. Biotechnol.Bioeng. 42 (2004) 643-656.

[9] J.L- Gouzé, G. Robledo, Feedback control for nonmonotone competition models in the chemostat. Nonlinear Analysis RWA. 6 (2005) 671-690.

[10] F. Grognard, F. Mazenc, A. Rapaport, Polytopic Lyapunov functions for persistence analysis of competing species. Discrete Contin. Dyn.Syst.Ser.B. 8 (2007) 73-93.

[11] H. Guo, S. Zheng, A competition model for two resources in un-stirred chemostat. Appl.Math.Comput. 217 (2011) 6934-6949.

[12] B. Haegeman, A. Rapaport, How flocculation can explain coexistence in the chemostat. J.Biol.Dyn. 2 (2008) 1-13.

[13] S.R. Hansen, S.P. Hubell, Single nutrient microbial competition: Agreement between experimental and forecast outcomes. Science. 207 (1980) 1491-1493.

[14] G. Hardin, Competitive exclusion principle. Science. 131 (1960) 1292-1297.

[15] J. Hofbauer, J.W.H. So, Competition in the gradostat: the global stability problem. Nonlinear Analysis TMA. 22 (1994) 1017-1031.

[16] J. Hofbauer, A unified approach to persistence. Acta.Appl.Math. 14 (1989) 14-22.

[17] S.B. Hsu, A competition model for a seasonally fluctuating nutrient. J.Math.Biol. 9 (1980) $115-132$

[18] S.B. Hsu, T. Hsu, Competitive exclusion of microbial species for a single-nutrient with internal storage. SIAM J.Appl.Math. 68 (2008) 1600-1617.

[19] S.B. Hsu, P. Waltman, On a system of reaction-diffusion equations arising from competition in an unstirred chemostat. SIAM J.Appl.Math. 53 (1993) 1026-1044.

[20] W.S. Keeran, P. De Leenheer, S. Pilyugin, Circular and elliptic orbits in a feedback-mediated chemostat. Discrete Contin. Dyn.Syst.Ser.B. 7 (2007) 779-792.

[21] T. Kuniya, Global stability analysis with a discretization approach for an age-structured multigroup SIR epidemic model. Nonlinear Analysis RWA. 12 (2011) 2640-2655.

[22] P. Lenas, S. Pavlou, Coexistence of Three Competing Microbial Populations in a Chemostat with Periodically Varying Dilution Rate. Math.Biosci. 129 (1995) 111-142.

[23] B. Li, H.L. Smith, Competition for essential resources: a brief review, in: Dynamical systems and its applications in biology. Fields Inst.Commun. 36 (2003) 213-227.

[24] C. Lobry, F. Mazenc, A. Rapaport, Persistence in ecological models of competition for a single resource. C.R. Math.Acad.Sci. Paris. 340 (2005) 199-204.

[25] P. Masci, F. Grognard, E. Benoît, O. Bernard, Competition between diverse types of microorganisms: exclusion and coexistence (submitted).

[26] F. Mazenc, M. Malisoff, J. Harmand, Further results on stabilization of periodic trajectories for a chemostat with two species. IEEE Trans.Aut.Contr. 53 (2008) 66-74.

[27] F. Mazenc, M. Malisoff, Remarks on output feedback stabilization of two-species chemostat models. Automatica. 46 (2010) 1739-1742.

[28] X. Meng, Z. Li, J.J. Nieto, Dynamic analysis of Michaelis-Menten chemostat-type competition models with time delay and pulse in a polluted environment. J.Math.Chem. 47 (2010) 123144.

[29] X. Meng, Q. Gao, Z. Li, The effects of delayed growth response on the dynamic behaviors of the Monod type chemostat model with impulsive input nutrient concentration. Nonlinear Analysis RWA. 11 (2010) 4476-4486.

[30] G. Meszéna, M. Gyllenberg, L. Pastor, J.A. Metz, Competitive exclusion and limiting similarity: a unified theory. Th.Pop.Biol. 69 (2006) 68-87.

[31] C. Neill, T. Daufresne, C.G. Jones, A competitive coexistence principle?. Oikos. 118 (2009) $1570-1578$.

[32] H. Nie, J. Wu, Coexistence of an unstirred chemostat model with Beddington-De Angelis functional response and inhibitor. Nonlinear Analysis RWA. 11 (2010) 3639-3652 (2010).

[33] S. Pavlou, Microbial competition in bioreactors. Chemical Industry and Chemical Engineering Quarterly, 12 (2006) 71-81.

[34] S. Pilyugin, P. Waltman, Competition in the unstirred chemostat with periodic input and washout. SIAM J.Appl.Math. 59 (1989) 1157-1177. 
[35] S. Pilyugin, P. Waltman, Multiple limit cycles in the chemostat with variable yield. Math.Biosci. 182 (2003) 151-166.

[36] E. Postma, A. Kuiper, W.F. Tomasouw, W.A. Scheffers, J.P. Van Dijken, Competition for glucose between the yeasts Saccharomyces cerevisiae and Candida utilis. Appl.Environ.Microbiol. 55 (1989) 3124-3220.

[37] H.L. Smith, Competitive coexistence in an oscillating chemostat. SIAM J.Appl.Math. 40 (1981) 498-522.

[38] H.L. Smith, P. Waltman, The Theory of the Chemostat. Dynamics of Microbial Competition, Cambridge University Press, Cambridge, 1995.

[39] I. Vatcheva, O. Bernard, H. De Jong, N. Mars, Experiment selection for the discrimination of semi-quantitative models of dynamical systems. Artif. Intel. 170 (2006) 472-506.

[40] M. Vidyasagar, Decomposition techniques for large--scale systems with nonadditive interactions: stability and stabilizability. IEEE Trans.Automat.Contr. AC-21 (1980) 773-779.

[41] L. Wang, L. Chen, J.J. Nieto, The dynamics of an epidemic model for pest control with impulsive effect. Nonlinear Analysis RWA. 11 (2010) 1374-1386.

[42] G.S.K. Wolkowicz, X-Q. Zhao, $n$-species competition in a periodic chemostat. Differential Integral Equations. 11 (1998) 465-491.

[43] H. Zhang, L. Chen, J.J Nieto, A delayed epidemic model with stage-structure and pulses for pest management strategy. Nonlinear Analysis RWA. 9 (2008) 1714-1726.

[44] H. Zhang, P. Georgescu, J.J. Nieto, L. Chen, Impulsive perturbation and bifurcation of solutions for a model of chemostat with variable yield. Appl.Math.Mech. 30 (2009) 933-944.

Universidad de Chile, Facultad de Ciencias, Departamento de Matemáticas. Las Palmeras 3425 Ñuñoa Santiago (CHILE)

Institut National de la Recherche en Informatique et Automatique INRIA, BIOCORE, 2004, Route des Lucioles, BP 93, 06902 Sophia Antipolis (France)

E-mail address: grobledo@uchile.cl,Frederic.Grognard@inria.fr, Jean-Luc.Gouze@inria.fr 